\title{
Oblique Closing Wedge Osteotomy and Lateral Plating for Cubitus Varus in Adults
}

\author{
Hyun Sik Gong MD, PhD, Moon Sang Chung MD, PhD, \\ Joo Han Oh MD, PhD, Hoyune Esther Cho, \\ Goo Hyun Baek MD, PhD
}

Received: 8 August 2007 / Accepted: 24 January 2008/Published online: 14 February 2008

(C) The Association of Bone and Joint Surgeons 2008

\begin{abstract}
Corrective osteotomy around the elbow can require longer recovery time in adults than in children because of the longer healing period and the propensity for stiffness. We hypothesized a lateral oblique closing wedge osteotomy with a larger contact area and fixation with a lag screw plus early motion would provide stable fixation and early motion recovery in adults with cubitus varus deformity. Twelve consecutive patients who needed surgery were treated using this procedure. They were allowed active motion exercises 1 week postoperatively. The age of the patients at the time of surgery averaged 39 years (range, 31-48 years). The minimum followup was 15 months. All patients achieved healing of the osteotomy, and regained preoperative arcs of elbow motion at a mean of 7.3 weeks (range, 2-12 weeks) postoperatively. The average humerus-elbow-wrist angle improved from $-23.3^{\circ}$ to $8^{\circ}(\mathrm{p}<0.002)$ by a mean gain of $29.6^{\circ}$. The mean lateral prominence index did not increase postoperatively. The final MEPI and DASH score averaged 95.4 points and 5.5 points. No patient experienced nerve palsy. Oblique osteotomy and fixation with a lag screw and lateral plating is a reasonable alternative technique for cubitus varus in adults,
\end{abstract}

Each author certifies that he has no commercial associations (eg, consultancies, stock ownership, equity interest, patent/licensing arrangements, etc) that might pose a conflict of interest in connection with the submitted article.

Each author certifies that his or her institution has approved the reporting of these cases, that all investigations were conducted in conformity with ethical principles of research, and that informed consent for participating in the study was obtained.

H. S. Gong ( $\varangle)$, M. S. Chung, J. H. Oh, H. E. Cho, G. H. Baek Department of Orthopaedic Surgery, Seoul National University Bundang Hospital, 300 Gumi-dong, Bundang-gu, Seongnam-si, Gyeonggi-do 463-707, Korea

e-mail: hsgong@snu.ac.kr with early recovery of elbow motion and satisfactory deformity correction.

Level of Evidence: Level IV, therapeutic study. See the Guidelines for Authors for a complete description of levels of evidence.

\section{Introduction}

Cubitus varus deformity is a common complication of pediatric supracondylar fracture of the humerus. Surgeons have proposed various surgical techniques for correction of the deformity including lateral closing wedge osteotomy $[1,3]$, medial open wedge osteotomy [11], dome osteotomy $[8,20,25]$, pentalateral osteotomy [14], three-dimensional osetotomy [2, 26], and step-cut translational osteotomy [10]. These techniques have emphasized primarily the osteotomy method that can correct three-dimensional deformity and reduce prominence of the lateral condyle. However, most of the previous studies on corrective osteotomy for cubitus varus have included young $[1,3,8,20$, 26] or mixed age groups [10,13,14, 16, 25] or used different fixation techniques in the same osteotomy method $[2,25]$.

Clinical experiences and animal studies indicate that fracture healing times differ among patients of different age groups [4, 15, 17]. For children with high healing capacity, union of a distal humeral osteotomy is achieved within 3 to 6 weeks and elbow function returns quickly [1, 20, 26]. In addition, children's elbows usually do not have the propensity for stiffness as those of adults [5, 21]. For adults, the union of the osteotomy or fracture repair in this region usually requires 3 months $[10,12]$. Although nonunion after corrective osteotomy was rarely reported, the incidence of delayed union or nonunion in the distal 
humerus fracture was reported to be as much as $10 \%$ [6]. The occurrence of posttraumatic stiffness in the adult elbow suggests the possibility of a longer recovery time after corrective osteotomy in adults. Literature on the corrective osteotomy for cubitus varus either included only a small number of adult patients [16, 25], or did not report how fast functional recovery was achieved by adult patients $[2,10,14]$.

A larger or longer screw has greater holding strength than a smaller or shorter screw, and properly placed lag screws increase fracture stability and lead to better fracture healing [27]. An oblique osteotomy increases the contact area at the osteotomy and allows lag screw fixation [23]. Lateral plating allows fixation with longer screws and can avoid olecranon osteotomy or division of the triceps muscle used for posterior plate fixation [10, 25], which has a risk of nonunion or muscle weakness.

We therefore hypothesized a lateral oblique closing wedge osteotomy with a larger contact area and fixation with a lag screw plus early motion would provide stable fixation and early motion recovery in adults with cubitus varus deformity. Our second hypothesis was that this osteotomy will reliably correct the deformity and prevent lateral prominence.

\section{Materials and Methods}

Twelve adult patients with cubitus varus deformity were treated with an oblique closing wedge osteotomy and fixation with a lag screw and lateral plating between May 2003 and June 2006. This study was performed under a protocol approved by our institutional review board. Data regarding physical and radiographic examinations were reviewed retrospectively using charts and radiographs. For functional assessments, patients were invited to our clinic.
All deformities resulted from malunion of distal humeral supracondylar fractures sustained by the patients when they were from 3 years to 8 years of age. There were five male and seven female patients. The average age at the time of surgery was 39 years (range, 31-48 years). The duration between the initial trauma and the corrective osteotomy averaged 33 years (range, 24-45 years). No patient had functional problems resulting from the deformity, such as ulnar nerve symptoms and posterolateral instability. However, all patients requested surgery for cosmetic reasons. Most of the patients stated that they have been reluctant to wear short sleeve shirts and their deformities have been affecting their social life.

Anteroposterior radiographs of both elbows with the elbows in full extension and supination were obtained before surgery. The humerus-elbow-wrist angle was measured on the anteroposterior radiographs (Fig 1A) [10]. We determined the extent of rotational deformity by physical examination in which the angle between the forearm and the back was measured with the elbow in $90^{\circ}$ flexion and the shoulder in hyperextension [29]. Range of motion of the elbow was measured while holding the medial and lateral condyle in the same horizontal plane to see the true flexion contracture of the elbow [10].

Preoperative varus deformity averaged $23^{\circ}$ (range, $8^{\circ}$ $44^{\circ}$ ). Internal rotation angle of the affected arm had a mean increase of $21^{\circ}$ (range, $10^{\circ}-30^{\circ}$ ) compared with the normal side. Except for three patients (Patients 6-8), all had nearly full range of motion preoperatively (Table 1).

We planned surgery using the Adobe Photoshop program (version 7.0; Adobe Systems, San Jose, CA). After determining the correction angle by comparing the humerus-elbow-wrist angle of the affected and the normal elbows, the wedge to be resected was drawn with its apex facing proximally. Then the distal part of the osteotomy was captured with the lasso tool and repositioned, and
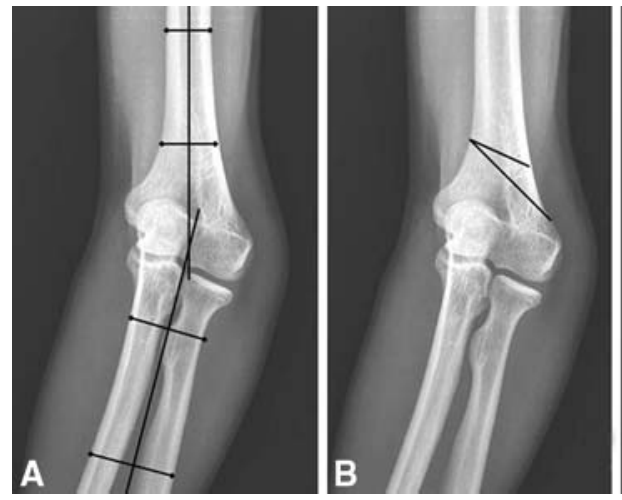

Fig. 1A-D (A) The humerus-elbow-wrist angle was measured, and surgical planning was performed using the Adobe Photoshop program (version 7.0; Adobe Systems, San Jose, CA). (B) The wedge to be resected was drawn with its apex facing proximally, and the angle of

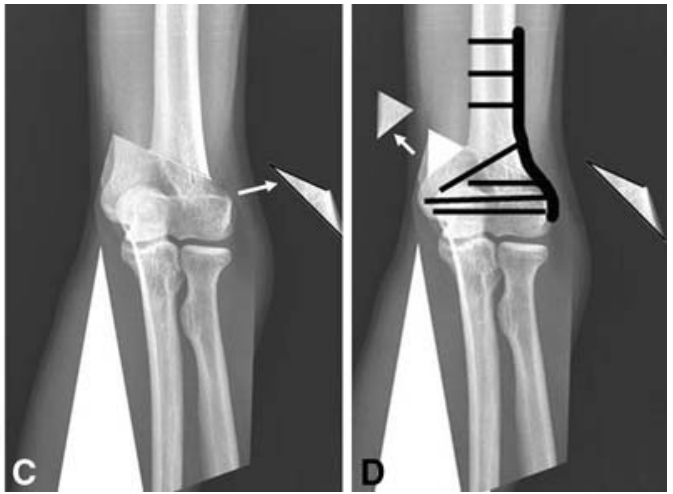

the wedge was identical to the desired correction angle. (C) The distal part of the osteotomy was repositioned. (D) Screws were drawn to simulate optimal fixation angle. 


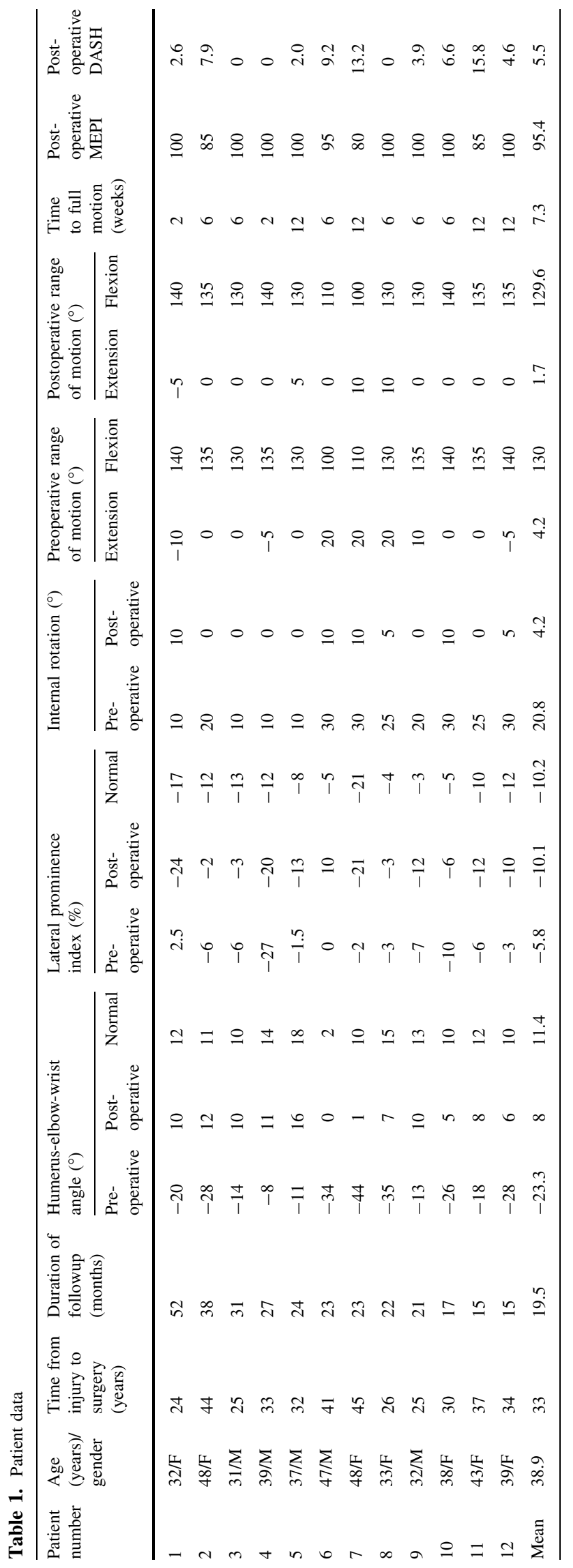

finally screws were drawn to simulate optimal fixation angle (Fig. 1).

We performed the operation with the patient in the lateral decubitus position. A posterior longitudinal skin incision was made in the posterior midline of the elbow. The ulnar nerve was released from the cubital tunnel and protected. We dissected the triceps muscle from the lateral cortex of the humerus and retracted it medially. An oblique closing wedge osteotomy was performed as planned using the Photoshop program. The angle of the wedge was identical to the desired correction angle. We repositioned the distal part of the osteotomy so the lateral cortices were continuous through the osteotomy to prevent prominence of the lateral condyle. Any internal rotational deformity was corrected using the lateral cortex as a hinge and rotating the distal part externally as much as measured preoperatively. We corrected any flexion deformity by simply excising more of the posterior part of the proximal osteotomy surface. The osteotomy was provisionally fixed with one or two Steinmann pins to check the humeruselbow-wrist angle and elbow motion. This step could be difficult as a result of the inherent instability of the oblique contact. The use of an additional large reduction clamp was helpful for temporary fixation. We performed final fixation by applying an anatomically designed, congruent plate (Acumed, Hillsboro, OR) and screws. We inserted three screws distally, three screws proximally, and one interfragmentary lag screw through the osteotomy to obtain compression. The prominent medial part was excised and the ulnar nerve was transposed anteriorly.

One week after surgery when the wound stabilized, the patients began active assistive range of motion exercises with intermittent protection of a long-arm removable splint. We instructed the patients to do one session of active assistive exercise for a minimum of 5 minutes per hour; therefore, doing 40 minutes of exercise after 8 of hours of sleep. Passive exercise was allowed 6 weeks and strengthening exercise was allowed 12 weeks postoperative. No formal therapy was administered and the splint usually was discarded by 2 to 6 weeks according to the patients' comfort.

All patients were followed up 2, 6, and 12 weeks after surgery and then every 6 months until 3 years after surgery. They also were invited to return for this study. The minimum followup was 15 months (mean, 26 months; range, 15-52 months). At each followup, we obtained radiographs of the elbow to check healing of the osteotomy, and we (HG) measured ranges of motion of the elbow by using a goniometer. A physician's assistant (SS) who was not directly involved in the care of the patients evaluated the outcome using the Mayo Elbow Performance Index (MEPI) [18], and obtained Disabilities of Arm, Shoulder, and Hand (DASH) outcome questionnaires for 


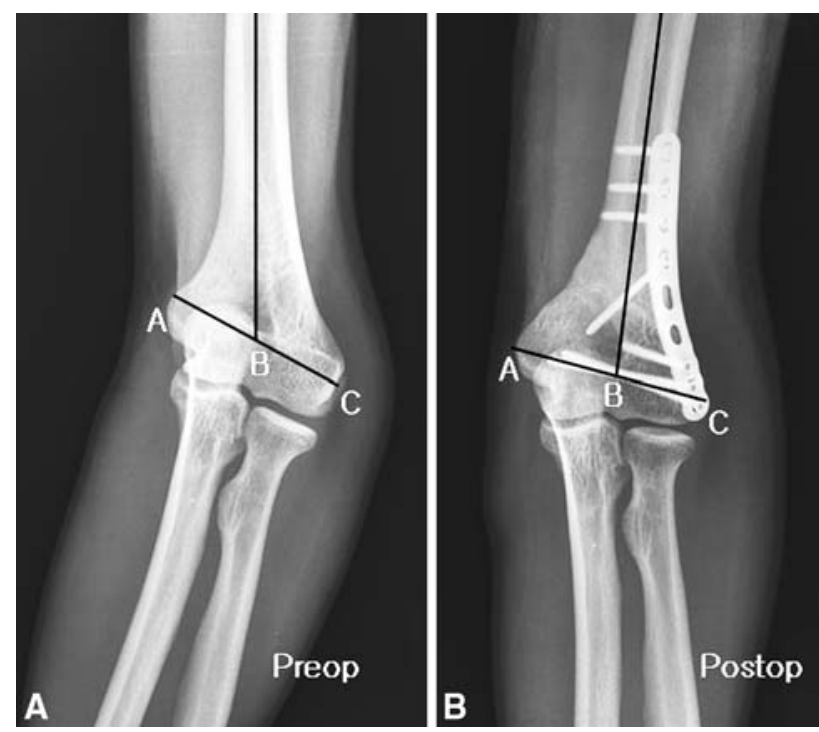

Fig. 2A-B The lateral prominence index (\%) was measured on the (A) preoperative and (B) postoperative radiographs using the formula, $(\mathrm{CB}-\mathrm{AB}) / \mathrm{AC} \times 100$. It is usually negative in normal elbows.

validated general assessment of upper extremity function $[7,24]$.

Using the radiographs obtained before surgery and those from the final followup, one author (HC) compared the humerus-elbow-wrist angles and the lateral prominence indices. The lateral prominence index was calculated using the method of Wong et al. [28], which is the difference between the measured medial and lateral widths of the bone from the longitudinal midhumeral axis and is expressed as a ratio of the total width of the distal humerus to minimize errors from magnification and individual variations of humeral size (Fig. 2).

Differences in the preoperative and postoperative radiographic values were determined with the Wilcoxon signed rank test. We used SPSS version 13.0 software (SPSS Inc, Chicago, IL).

\section{Results}

Our primary hypothesis was that a lateral oblique closing wedge osteotomy with a larger contact area and fixation with a lag screw plus early motion would provide stable fixation and early motion recovery. By using our rehabilitation protocol, all patients regained preoperative arcs of elbow motion at a mean of 7.3 weeks (range, 2-12 weeks) postoperatively. The range of motion of the forearm rotation did not change postoperatively. Clinical healing of the osteotomy was achieved by all 12 patients within 12 weeks of the operation. The time to early radiographic healing was not definite because callus did not form at the osteotomy site in most cases, indicating the nature of direct bone healing usually occurring in compression osteosynthesis [22].

Our second hypothesis was that this osteotomy would reliably correct the deformity and prevent lateral prominence. The average humerus-elbow-wrist angle improved from $-23.3^{\circ}$ to $8^{\circ}(\mathrm{p}<0.002)$ by a mean gain of $29.6^{\circ}$ (range, $19^{\circ}-45^{\circ}$ ). In 10 elbows, the difference of the humerus-elbow-wrist angle between the affected and the normal elbow was less than $5^{\circ}$, and in two elbows it was between $6^{\circ}$ and $10^{\circ}$. Those two patients had preoperative humerus-elbow-wrist angles of $-44^{\circ}$ and $-35^{\circ}$, and after correction of $45^{\circ}$ and $42^{\circ}$ by corrective osteotomy, they still had undercorrections of $9^{\circ}$ and $8^{\circ}$ compared with the normal side of $10^{\circ}$ and $15^{\circ}$. No elbow had residual varus deformity. The postoperative lateral prominence index averaged $-10.1 \%$ (range, $-24 \%-10 \%$ ) and the normal side had an average index of $-10.2 \%$ (range, $-21 \%--3 \%$ ). Compared with the mean preoperative value of $-5.8 \%$ (range, $-27 \%$ $2.5 \%$ ), the mean lateral prominence index did not increase after correction of the deformity (Table 1). All maintained a negative value of the lateral prominence index except one patient (Patient 6) who had an increase of $10 \%$.

The recent MEPI score averaged 95.4 points (range, 80 100 points), and the mean DASH score was 5.5 points (range, 0-15.8 points), both of which were consistent with excellent outcome [7, 18]. No patient experienced nerve palsies, instability, or wound problems, and no patient needed removal of the plate. One patient complained of prominent scar hypertrophy on the posterior side of the elbow. Another patient had limb shortening of approximately $1 \mathrm{~cm}$ because the sleeve was that much longer than before surgery.

\section{Discussion}

We hypothesized a lateral oblique closing wedge osteotomy with a larger contact area and fixation with a lag screw plus early motion would provide stable fixation and early motion recovery, with reliable correction of the cubitus varus deformity in adult patients. The data show all patients obtained cosmetically acceptable correction of the deformity and regained preoperative range of motion by at least 3 months postoperatively. The strength of the study design was that the rate of recovery of range of motion is reported. The strength of our procedure was that preoperative motion was recovered early in all patients without any serious complications.

There are several limitations in this study. We had only a small number of cases and we had no comparative osteotomy groups, although the outcomes were promising. We had no mechanical data to support the early motion exercise although it empirically worked. Radiographic 


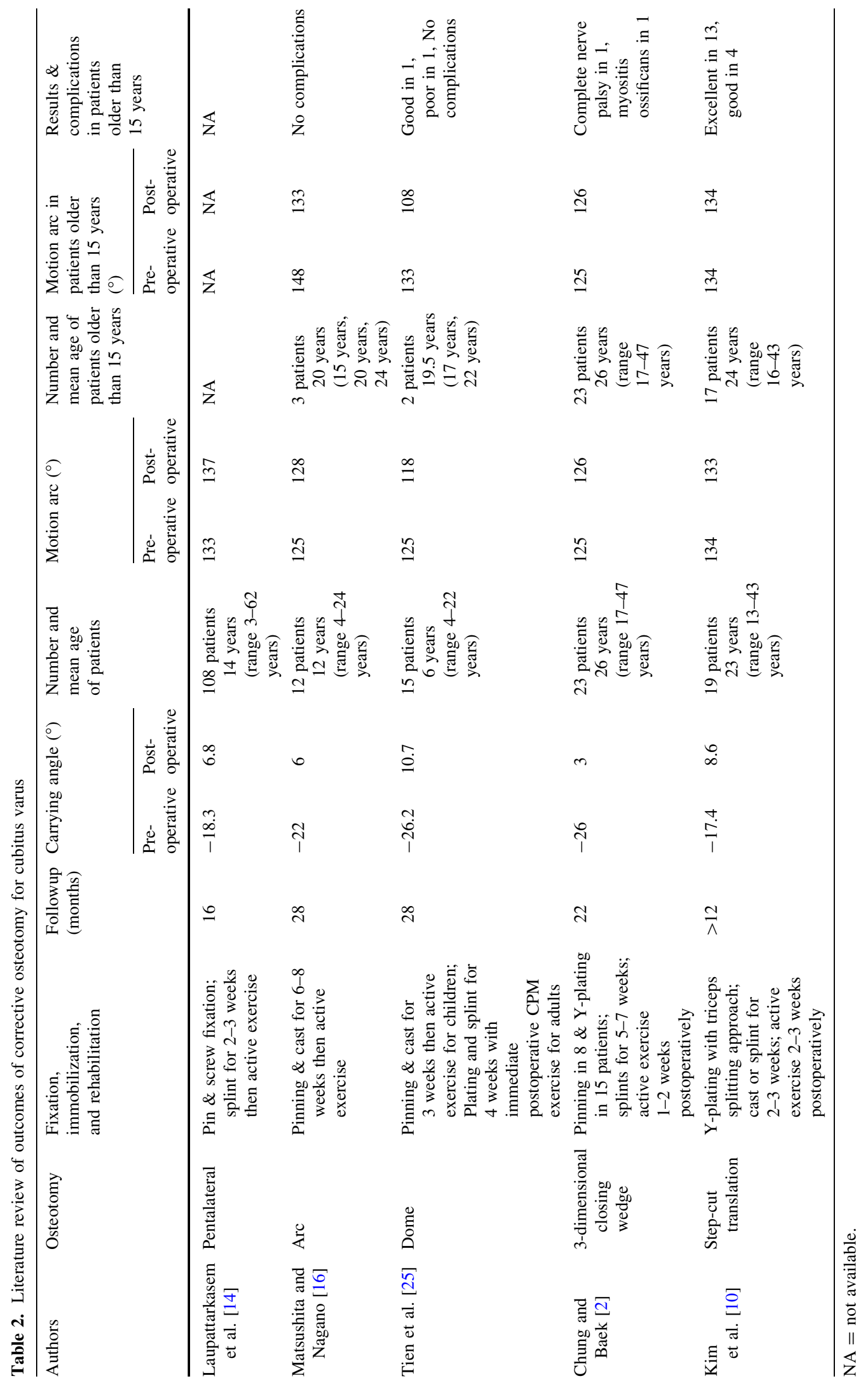


measurements were subject to intraobserver or interobserver variations, and reliability tests were not performed. However, one well-trained individual did the measurement using our institution's digitalized computer imaging system. Another limitation is the lack of preoperative functional assessment using the MEPI or DASH. However, as the patients had minimal preoperative functional disabilities, the results from MEPI or DASH would not be significant indicators of how effective surgical treatments were. This is proven by a high percentage of perfect scores as they indicate the ceiling effect. The MEPI and DASH may be better suited to represent postoperative functional status.

There are several studies reporting the outcome of corrective osteotomy for cubitus varus in adults (Table 2). Pentalateral osteotomy [14] was used for 108 patients at the mean age of 14 years with satisfactory results, but information on patients older than 15 years was lacking. Arc osteotomy [16] was used in three postpubertal patients with the mean age of 20 years, with pinning and 6 to 8 weeks of cast immobilization. In two of the three patients, total arc of motion decreased: in one patient by $40^{\circ}$ and in the other by $10^{\circ}$. In a study of the dome osteotomy by Tien et al. [25], olecranon osteotomy was done for two postpubertal patients to apply the plate posteriorly, and these patients lost motion by $20^{\circ}$ to $30^{\circ}$. Three-dimensional osteotomy [2] was done for 23 adult patients, with one nerve palsy associated with pinning and one myositis ossificans. In a step-cut osteotomy [10], the triceps muscle was divided to apply the Y-plate posteriorly. Although the triceps-splitting approach has been used safely for many elbow surgeries, peak triceps torque does not recover fully and the deficit is still $3 \%$ to $6 \%$ at 3 years and 6 months (3.5 years) after triceps division [9]. The average ages of patients in these studies were all less than 26 years, much younger than that of patients in our study (39 years). Our results were comparable or superior to those reported in the literature.

We suggest several advantages of this technique in comparison to other osteotomies and fixation methods. First, the oblique osteotomy can provide a larger contact surface of cancellous bone than a step-cut translational [10], transverse closing wedge [2], or dome osteotomy [8, $20,25]$ in a simple way (Figs. 3,4). In the step-cut translational osteotomy [10], the cortical surface of the lateral supracondylar ridge contacts the osteotomy site after translation and this may delay bone healing considering the fact that the lateral side is usually the tension side in daily activities [19]. Second, the lag screw allows compression of the osteotomy site. Although the concepts of bone healing are still evolving and biologic internal fixation is more appreciated [22], under similar open osteotomy techniques involving soft tissue stripping, optimal stability achieved by a lag screw will be beneficial in early motion exercise. No callus formation in most of
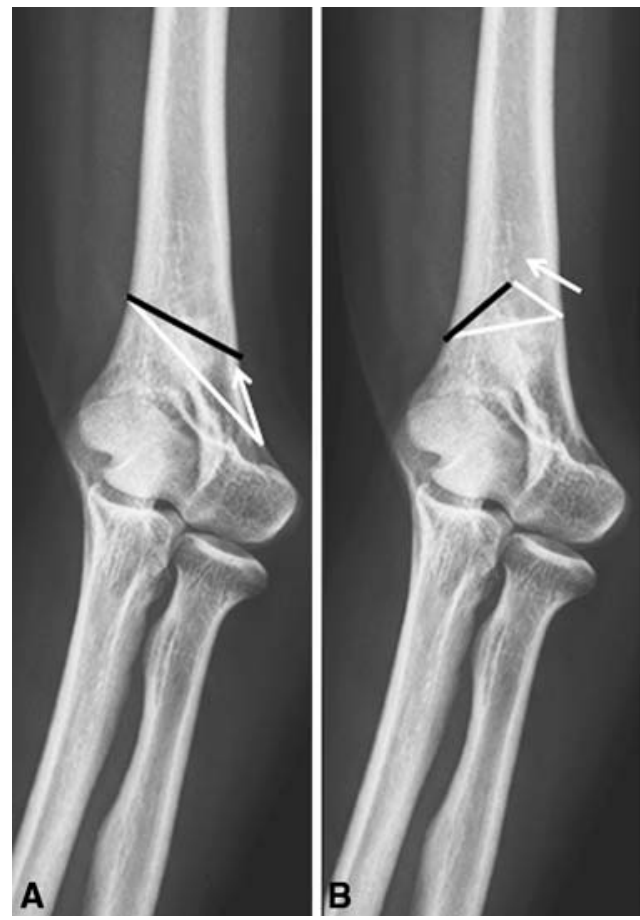

Fig. 3A-D Different types of osteotomies are shown based on the preoperative radiographs of a patient with cubitus varus deformity. The arrow indicates translocation of the distal part after bone cutting,

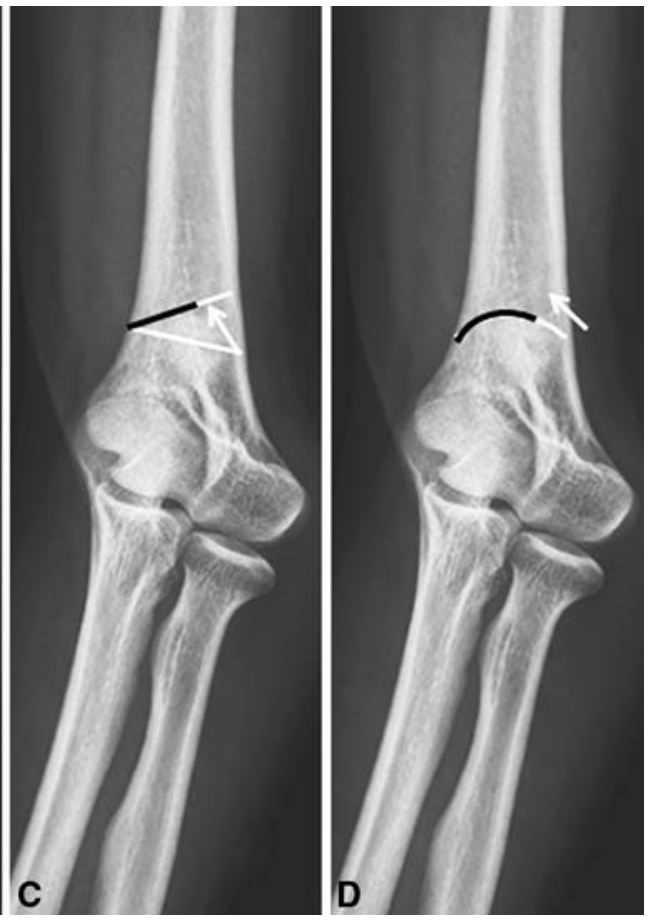

and the black line indicates contact surfaces of cancellous bone after (A) an oblique closing wedge, (B) a step-cut translational, (C) a transverse closing wedge, and (D) a dome osteotomy. 

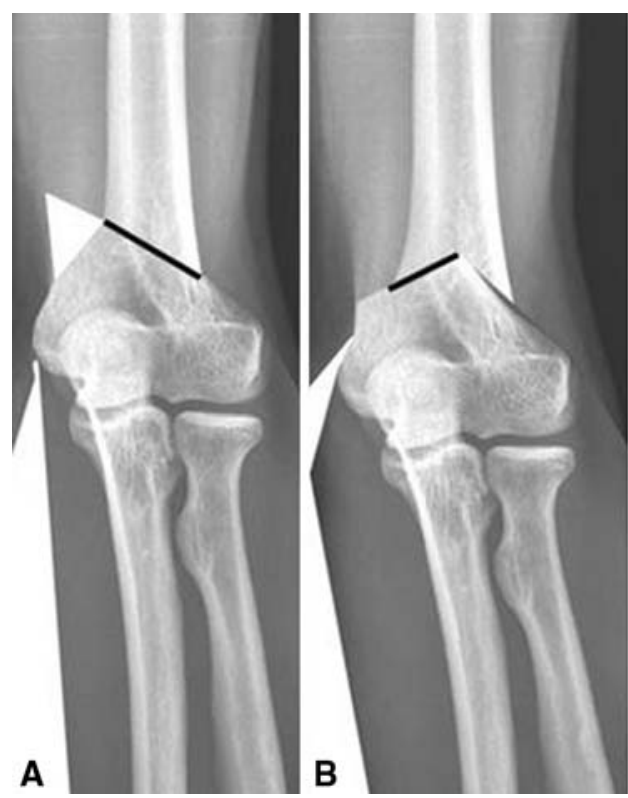

Fig. 4A-D Simulated postoperative radiographs after different types of osteotomy show that (A) the oblique closing wedge osteotomy has a larger cancellous bone contact surface than (B) a step-cut

the patients in this study indicates the stability of the fixation. Moreover, the lag screw direction from proximallateral to distal-medial seems to have a biomechanical advantage over the distal-lateral to proximal-medial direction [14] because the lateral cortex is the tension side and this may cause pullout of the screw when inserted from distal-lateral to proximal-medial. Third, the lateral plating allows fixation with longer screws according to the geometry of the distal humerus. A larger or longer screw will have greater holding strength than a smaller or shorter screw [27]. The lateral plating and the simplicity of the osteotomy also avoid olecranon osteotomy or division of the triceps muscle, which has a risk of nonunion, intraarticular or muscular adhesion, or weakness of the triceps muscle. Finally, the anatomically precontoured plate naturally translates the distal fragment medially. To apply the plate laterally, the lateral cortices should be continuous; this requires adequate medial translation and prevents prominence of the lateral condyle.

The disadvantage of this technique is that bone resection is larger than with the step-cut translational [10], transverse closing wedge [2], or dome osteotomy (Fig. 4) [8, 20, 25]. In terms of bone resection, dome osteotomy has no loss of bone and maintains the length of the humerus. In our experience, shortening of the humerus was not detectable clinically in most cases after correction of the deformity. Another disadvantage is when large correction of the rotational deformity is required, bony contact of this osteotomy can be compromised because this technique uses the lateral cortex as a hinge and this may cause no contact of the osteotomy surface on the medial side.

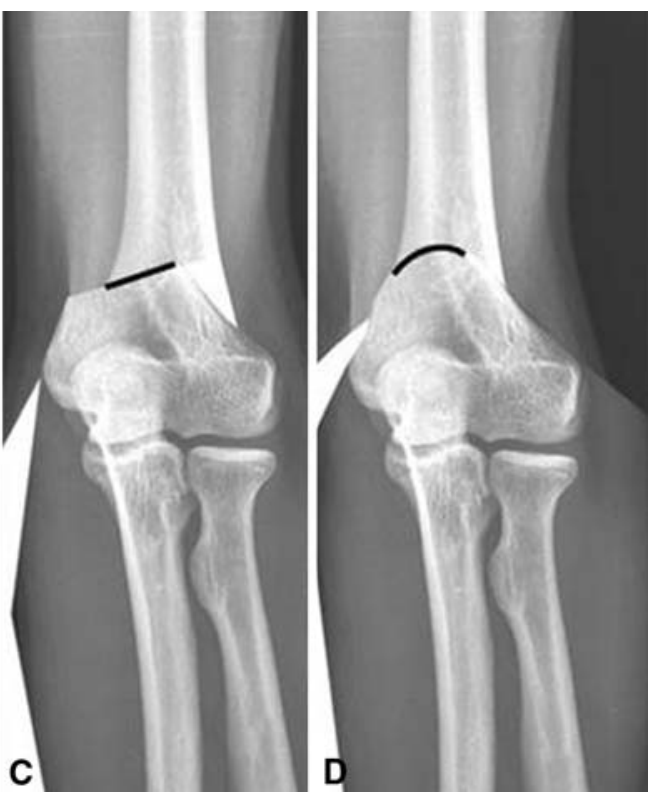

translational, (C) a transverse closing wedge, and (D) a dome osteotomy. Joint line elevation is also largest in the oblique closing wedge osteotomy, indicating shortening of the humerus.

However, all osteotomy techniques have the same difficulty when any rotation is required at the time of correction because of the thin cortex of the distal humerus. We believe additional short posterior plating or bone grafting may be necessary when a large amount of rotational correction is needed.

With the limitations mentioned, an oblique osteotomy and fixation by a lag screw and lateral plating can be a reasonable alternative for correction of a cubitus varus deformity in adult patients, with satisfactory deformity correction, reliable healing of the osteotomy, and early functional recovery.

Acknowledgments We thank Sang Mi Shim and Dr Sang Ik Shin for their skillful contributions to this study.

\section{References}

1. Bellemore MC, Barrett IR, Middleton RW, Scougall JS, Whiteway DW. Supracondylar osteotomy of the humerus for correction of cubitus varus. J Bone Joint Surg Br. 1984;66: $566-572$.

2. Chung MS, Baek GH. Three-dimensional corrective osteotomy for cubitus varus in adults. J Shoulder Elbow Surg. 2003;12:472-475.

3. Devnani AS. Lateral closing wedge supracondylar osteotomy of humerus for post-traumatic cubitus varus in children. Injury. 1997;28:643-647.

4. Gruber R, Koch H, Doll BA, Tegtmeier F, Einhorn TA, Hollinger JO. Fracture healing in the elderly patient. Exp Gerontol. 2006;41:1080-1093.

5. Hart GM, Wilson DW, Arden GP. The operative management of the difficult supracondylar fracture of the humerus in the child. Injury. 1977;9:30-34. 
6. Helfet DL, Kloen P, Anand N, Rosen HS. Open reduction and internal fixation of delayed unions and nonunions of fractures of the distal part of the humerus. J Bone Joint Surg Am. 2003;85:33-40.

7. Hudak PL, Amadio PC, Bombardier C. Development of an upper extremity outcome measure: the DASH (disabilities of the arm, shoulder and hand). The Upper Extremity Collaborative Group (UECG). Am J Ind Med. 1996;29:602-608.

8. Kanaujia RR, Ikuta Y, Muneshige H, Higaki T, Shimogaki K. Dome osteotomy for cubitus varus in children. Acta Orthop Scand. 1988;59:314-317.

9. Kasser JR, Richards K, Millis M. The triceps-dividing approach to open reduction of complex distal humeral fractures in adolescents: a Cybex evaluation of triceps function and motion. J Pediatr Orthop. 1990;10:93-96.

10. Kim HT, Lee JS, Yoo CI. Management of cubitus varus and valgus. J Bone Joint Surg Am. 2005;87:771-780.

11. King D, Secor C. Bow elbow (cubitus varus). J Bone Joint Surg Am. 1951;33:572-576.

12. Kinik H, Atalar H, Mergen E. Management of distal humerus fractures in adults. Arch Orthop Trauma Surg. 1990;119:467-469.

13. Kumar K, Sharma VK, Sharma R, Maffulli N. Correction of cubitus varus by French or dome osteotomy: a comparative study. J Trauma. 2000;49:717-721.

14. Laupattarakasem W, Mahaisavariya B, Kowsuwon W, Saengnipanthkul S. Pentalateral osteotomy for cubitus varus: clinical experiences of a new technique. J Bone Joint Surg Br. 1989;71:667-670.

15. Lu C, Miclau T, Hu D, Hansen E, Tsui K, Puttlitz C, Marcucio RS. Cellular basis for age-related changes in fracture repair. J Orthop Res. 2005;23:1300-1307.

16. Matsushita T, Nagano A. Arc osteotomy of the humerus to correct cubitus varus. Clin Orthop Relat Res. 1997;336:111-115.

17. Meyer RA Jr, Tsahakis PJ, Martin DF, Banks DM, Harrow ME, Kiebzak GM. Age and ovariectomy impair both the normalization of mechanical properties and the accretion of mineral by the fracture callus in rats. J Orthop Res. 2001;19:428-435.
18. Morrey BF, An KN, Chao EY. Functional evaluation of the elbow. In: Morrey BF, ed. The Elbow and Its Disorders. 2nd ed. Philadelphia, PA: WB Saunders Company; 1993:86-97.

19. O'Driscoll SW. Optimizing stability in distal humeral fracture fixation. J Shoulder Elbow Surg. 2005;14(suppl S):186S-194S.

20. Pankaj A, Dua A, Malhotra R, Bhan S. Dome osteotomy for posttraumatic cubitus varus: a surgical technique to avoid lateral condylar prominence. J Pediatr Orthop. 2006;26:61-66.

21. Papandrea R, Waters PM. Posttraumatic reconstruction of the elbow in the pediatric patient. Clin Orthop Relat Res. 2000; 370:115-126.

22. Perren SM. Evolution of the internal fixation of long bone fractures. The scientific basis of biological internal fixation: choosing a new balance between stability and biology. J Bone Joint Surg Br. 2002;84:1093-1110.

23. Rayhack JM. Ulnar shortening. Tech Hand Up Extrem Surg. 2003;7:52-60.

24. SooHoo NF, McDonald AP, Seiler JG 3rd, McGillivary GR. Evaluation of the construct validity of the DASH questionnaire by correlation to the SF-36. J Hand Surg Am. 2002;27: 537-541.

25. Tien YC, Chih HW, Lin GT, Lin SY. Dome corrective osteotomy for cubitus varus deformity. Clin Orthop Relat Res. 2000;380: $158-166$

26. Uchida Y, Ogata K, Sugioka Y. A new three-dimensional osteotomy for cubitus varus deformity after supracondylar fracture of the humerus in children. J Pediatr Orthop. 1991;11:327-331.

27. Uhl RL. The biomechanics of screws. Orthop Rev. 1989; 18:1302-1307.

28. Wong HK, Lee EH, Balasubramaniam P. The lateral condylar prominence: a complication of supracondylar osteotomy for cubitus varus. J Bone Joint Surg Br. 1990;72:859-861.

29. Yamamoto I, Ishii S, Usui M, Ogino T, Kaneda K. Cubitus varus deformity following supracondylar fracture of the humerus: a method for measuring rotational deformity. Clin Orthop Relat Res. 1985;201:179-185. 